\title{
Reparación laparoscópica de hernia de Spiegel con colocación de malla compuesta intraperitoneal y sistema de fijación articulado. Reporte de dos casos
}

\author{
Laparoscopic Spigelian hernia repair with intraperitoneal onlay composite mesh and \\ articulated fixing system. Two cases report
}

\section{Ernesto A. Dzib-Calan ${ }^{1 *}$, Sergio F. Ortiz-Reyes², Jorge I. Morales-Pérez ${ }^{1}$, Rodrigo Núñez-Vidales², Gerardo Rodarte-Cajica² y Gustavo Leal-Mérida ${ }^{3}$}

${ }^{1}$ Departamento de Cirugía, Escuela de Posgrado de Sanidad Naval; ${ }^{2}$ Servicio de Cirugía General; ${ }^{3}$ Área Quirúrgica. Hospital General Naval de Alta Especialidad, Ciudad de México, México

\section{Resumen}

Introducción: La hernia de Spiegel es un tipo infrecuente de defecto de la pared abdominal. Tradicionalmente se realizaba su reparación con técnica abierta, pero en la actualidad contamos con múltiples técnicas laparoscópicas con uso de material protésico. Caso clínico: Presentamos dos casos de hernia de Spiegel, un paciente programado de manera electiva y otro de urgencia por hernia incarcerada, ambos diagnosticados de manera prequirúrgica con tomografía abdominal. Discusión: Los dos pacientes fueron intervenidos de manera exitosa por vía laparoscópica con técnica de malla intraperitoneal utilizando malla compuesta y sistema de fijación articulado. Conclusiones: Esta técnica se muestra como una opción recomendable por su facilidad de realización y buenos resultados, tanto para cirugías electivas como para cirugías de urgencia.

PALABRAS CLAVE: Hernia. Laparoscopia. Spiegel.

\begin{abstract}
Introduction: Spiegel hernia is an infrequent type of abdominal wall defect. Traditionally, it was repaired with an open technique, currently we have multiple laparoscopic techniques with the use of prosthetic material. Clinical case: We present two cases of Spiegel hernia, one patient scheduled electively and one patient of urgency due to incarcerated hernia, both diagnosed pre-surgically with abdominal tomography. Discussion: Both patients were successfully operated laparoscopically with intraperitoneal onlay mesh technique using composite mesh and articulated fixation system. Conclusion: This technique is shown as a recommended option due to its technical facility, good results, both for elective surgeries and emergency surgeries.
\end{abstract}

KEY WORDS: Hernia. Laparoscopic. Spiegel.

\footnotetext{
Correspondencia:

*Ernesto A. Dzib-Calan

Av. Heroica Escuela Naval Militar, edif. 1, depto. 301 


\section{Introducción}

La hernia de Spiegel es un tipo de hernia poco frecuente de la pared abdominal, situada en la unión del borde lateral del músculo recto del abdomen y el borde medial del transverso del abdomen, la mayoría en la línea semilunar (línea de Spiegel). El saco herniario penetra a través de la aponeurosis del músculo transverso del abdomen y el músculo oblicuo interno, pero permanece por detrás de la aponeurosis del músculo oblicuo externo ${ }^{1-3}$.

La hernia de Spiegel representa el 0.12 al $2 \%$ de las hernias de la pared abdominal, con un pico de incidencia entre los 50 y los 60 años de edad ${ }^{1,4}$.

Los pacientes pueden cursar asintomáticos 0 presentar datos inespecíficos, como dolor abdominal o sensación de una masa en los cuadrantes inferiores del abdomen. El diagnóstico clínico puede ser difícil de realizar, principalmente en pacientes obesos. Dentro de los estudios de imagen, el ultrasonido es económico y de gran precisión en el diagnóstico. La tomografía nos ayuda a establecer el diagnóstico con alta sensibilidad y especificidad, pero con las desventajas de su disponibilidad y costo ${ }^{5}$.

Inicialmente, la hernia de Spiegel era reparada mediante un procedimiento de cirugía abierta. En la actualidad, con la llegada de la laparoscopia, han surgido diversas técnicas que disminuyen las tasas de recidiva, el dolor posoperatorio y el periodo de convalecencia posquirúrgica, y que mejoran los resultados estéticos ${ }^{6,7}$. Entre las técnicas más utilizadas se encuentran la totalmente extraperitoneal (TEP), la transabdominal preperitoneal (TAPP) y la colocación de una malla intraperitoneal superpuesta (IPOM, intraperitoneal onlay mesh), e incluso se ha descrito su reparación asistida por robot en casos aislados reportados en la literatura ${ }^{8-10}$. Ninguna de las anteriores se ha considerado como método de referencia para el manejo de este tipo de hernia.

Nosotros presentamos dos casos de hernia de Spiegel sintomática que fueron resueltos de manera exitosa con la técnica IPOM y con uso de un sistema de fijación articulado.

\section{Casos clínicos}

\section{Paciente 1}

Mujer de 74 años con antecedentes quirúrgicos de oclusión tubárica bilateral, artroplastia total de rodilla

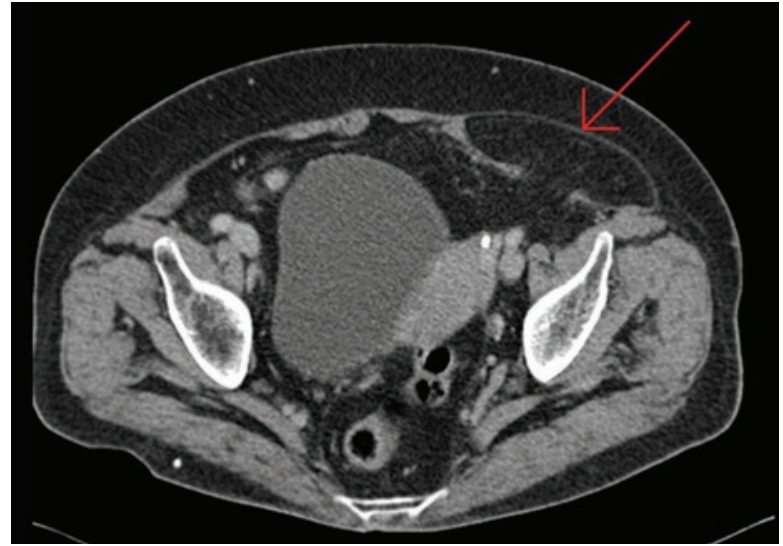

Figura 1. Imagen de tomografía abdominal que muestra una hernia de Spiegel lateral izquierda no complicada.

derecha y artroscopia bilateral; niega trastornos crónicos degenerativos. Presenta un cuadro de 2 años de evolución con aumento de volumen en la fosa ilíaca izquierda acompañado de dolor de moderada intensidad, el cual se exacerba con los esfuerzos físicos; niega alteraciones del hábito intestinal y otra sintomatología. A la exploración física, el abdomen es globoso a expensas de panículo adiposo, con dolor a la palpación media en la fosa ilíaca izquierda y un abultamiento mal delimitado, blando, fijo y no reducible; no se palpa defecto herniario, que se evidencia más con la maniobra de Valsalva. Se realiza tomografía simple, la cual reporta un defecto aponeurótico de $22 \times 33 \mathrm{~mm}$ lateral al músculo recto anterior izquierdo en la fascia posterior y contenido por la fascia anterior del músculo oblicuo externo, saco herniario de $80 \times 33 \mathrm{~mm}$ contenido en el mesenterio y el ligamento redondo homolateral. Impresión diagnóstica de hernia de Spiegel no complicada (Fig. 1). Se realiza cirugía laparoscópica con técnica intraperitoneal, en un tiempo quirúrgico total de 35 minutos, con un sangrado de $5 \mathrm{ml}$. La paciente cursa con buena evolución, dolor posoperatorio leve, sin ninguna complicación, y se egresa al día siguiente. Se da seguimiento por la consulta externa hasta 3 meses sin que apaezcan recurrencia, dolor ni formación de seroma.

\section{Paciente 2}

Varón de 87 años con antecedente de cardiopatía isquémica, revascularización aortocoronaria hace 20 años, operado de apendicectomía abierta, bajo tratamiento con ácido acetilsalicílico, clopidogrel y espironolactona, y con hipertensión arterial sistémica sin manejo. Con el antecedente de un aumento de volumen en la fosa ilíaca izquierda, el cual se reduce 


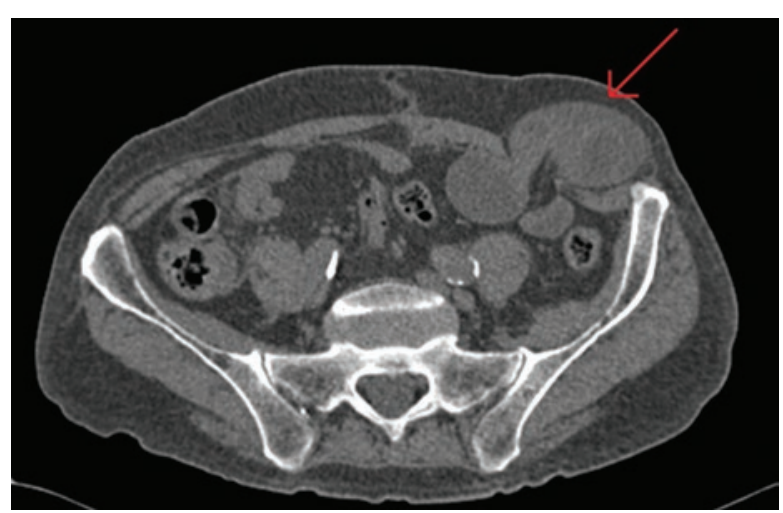

Figura 2. Tomografía abdominal en la que se aprecia una hernia de Spiegel lateral izquierda con incarceramiento de asas intestinales.

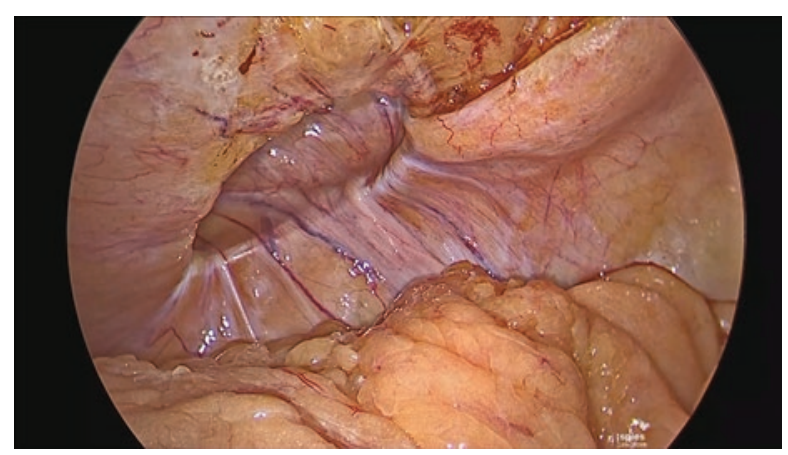

Figura 3. Defecto herniario (paciente 1).

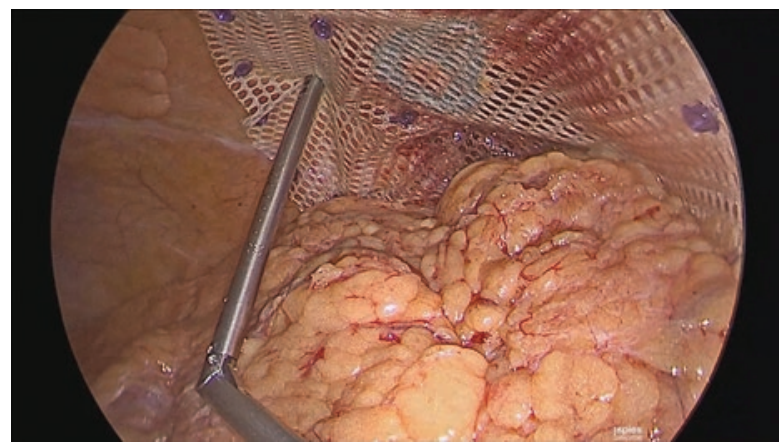

Figura 4. Fijación de la malla con sistema articulado (paciente 1).

de manera espontánea, acompañado de dolor leve ocasional y exacerbación con la realización de esfuerzos físicos, acude al servicio de urgencias por presentar dolor de gran intensidad e incapacidad de reducir el abultamiento en la fosa iliaca izquierda, así como náuseas y vómitos. A la exploración se observa un abultamiento de aproximadamente $6 \times 6 \mathrm{~cm}$, doloroso a la palpación y no reducible; no se palpa defecto herniario. Se realiza tomografía abdominopélvica que reporta defecto de la pared abdominal de 20 $\times 30 \mathrm{~mm}$, con saco de $40 \times 60 \mathrm{~mm}$, protrusión de grasa peritoneal y asas intestinales en la interlínea del músculo recto abdominal y del oblicuo abdominal izquierdo, con impresión diagnóstica de hernia de Spiegel incarcerada (Fig. 2). Se realiza cirugía con abordaje laparoscópico y se encuentra un incarceramiento de asas de intestino, que se reducen de manera exitosa y conservan su viabilidad. Posteriormente se hace la reparación con técnica intraperitoneal en un tiempo quirúrgico de 44 minutos, con un sangrado de $5 \mathrm{ml}$. El paciente cursa el posquirúrgico sin complicaciones y se mantiene en vigilancia por 48 horas debido a su comorbilidad; posteriormente es egresado con seguimiento por consulta externa hasta los 3 meses, sin observar datos de recurrencia ni complicaciones tardías.

\section{Técnica quirúrgica}

Con el paciente bajo anestesia general, en decúbito supino, con ambos brazos asegurados a los lados, se inicia el neumoperitoneo a $12 \mathrm{mmHg}$ con aguja de Veress para posterioremente colocar un trocar de $10 \mathrm{~mm}$ en la línea media clavicular contralateral a la hernia a nivel de la cicatriz umbilical. Se realiza laparoscopia diagnóstica en busca de sangrado o lesión de órganos durante el abordaje, y se colocan dos trocares de $5 \mathrm{~mm}$ bajo visión directa superior e inferior al primer trocar a lo largo de la línea media clavicular. Se procede a identificar el sitio de la hernia y se realiza la disección de las adherencias con pinza Ligasure ${ }^{\mathrm{TM}}$ Blunt hasta la reducción del contenido y la visualización del orificio herniario. Para el cierre del defecto se opta por una malla compuesta Symbotex ${ }^{\mathrm{TM}}$ composite mesh, de monofilamento de polipropileno con un recubrimiento de colágeno y con unas medidas de $15 \times 10 \mathrm{~cm}$, que se introduce por el trocar de $10 \mathrm{~mm}$ previamente humidificada y se sitúa sobre el defecto, al que se adhiere de manera momentánea por encontrarse húmedo. Posteriormente se realiza la fijación de la malla con aplicador de tacks tipo tornillo ReliaTack ${ }^{\mathrm{TM}}$, que es un sistema de fijación articulado que permite para su aplicación un ángulo de hasta $65^{\circ}$ y con ello se facilita la aplicación de los tacks en ángulos difíciles de alcanzar en la pared abdominal, como es el caso de las hernias ventrales y de Spiegel (Figs. 3 a 6). Se verifica la adecuada hemostasia en la cavidad y se extraen los trocares bajo visión directa para descartar el sangrado en el sitio de inserción.

\section{Discusión}

Las hernias de Spiegel son un tipo de hernia infrecuente y subdiagnosticada, lo que ha ocasionado que 


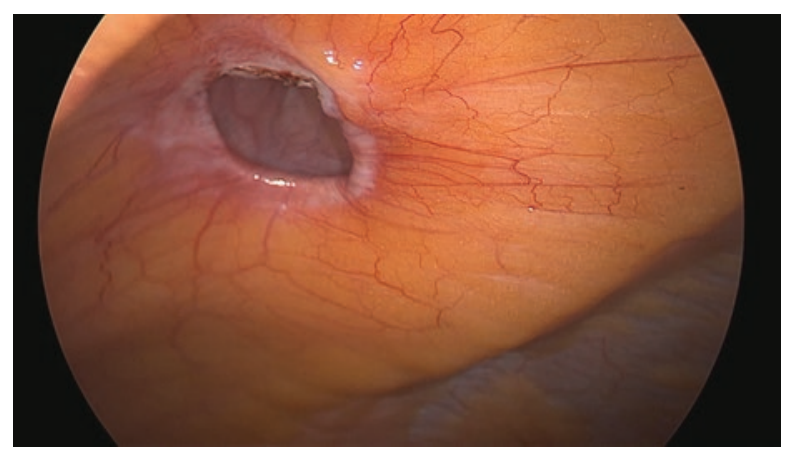

Figura 5. Defecto herniario (paciente 2).

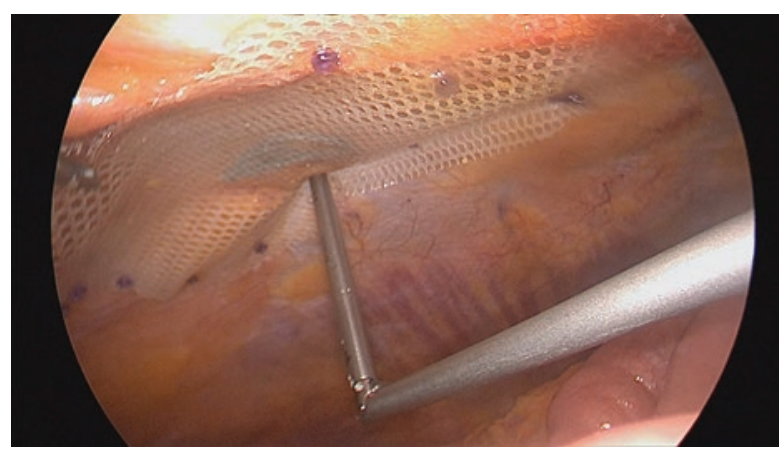

Figura 6. Colocación de la malla compuesta (paciente 2).

no se tenga una técnica quirúrgica considerada de manera preferente en este tipo de hernias. Se han descrito numerosas técnicas, desde la cirugía abierta con tensión hasta la introducción de la cirugía laparoscópica en 1992, detallada por primera vez por Carter y Mizes ${ }^{11}$, y actualmente asistida con robot, con uso de mallas protésicas de diversos tipos; sin embargo, en su mayoría son reportes de casos o pequeñas series de pacientes en los que incluso se reportan las hernias de Spiegel como diagnóstico incidental durante otro procedimiento.

El métodos con más frecuencia referido en la literatura es IPOM, seguido de TAPP y por último TEP'2.

La técnica IPOM es considerada la más popular y frecuente, principalmente por ser más fácil de realizar, lo que se traduce en una menor curva de aprendizaje y una disminución de los tiempos quirúrgicos, pero con la desventaja de entrar a la cavidad peritoneal y, al estar el material protésico en contacto con vísceras, el riesgo de inflamación y de formación de adherencias y seromas ${ }^{13}$.

La TAPP ofrece, al igual que la IPOM, la ventaja de la visualización del contenido del saco herniario y la viabilidad de este en el caso de hernias incarceradas, con el beneficio de colocar la malla preperitoneal sin contacto con las asas intestinales, pudiendo realizar el cierre del defecto y la posterior colocación de la malla.
La TEP es la técnica de mayor dificultad con respecto a las anteriores, por lo que requiere ser realizada por un cirujano experto en ella. Tiene la ventaja de no entrar a la cavidad abdominal y así disminuye el riesgo de daño al intestino, pero con la incapacidad de explorar el contenido del saco herniario, por lo que se recomienda en pacientes programados de manera electiva y no para aquellos que ingresan por urgencias ante un escenario de hernia incarcerada 0 estrangulada ${ }^{14}$.

Se han descrito otras técnicas, como la SILTEP (single incision laparoscopic totally extraperitoneal), principalmente utilizada para reparación de hernia inguinal, con la cual se ha podido resolver de manera exitosa una hernia de Spiegel encontrada incidentalmente y diagnosticada de manera preoperatoria ${ }^{15}$; sin embargo, este procedimiento no ofrece la ventaja de la inspección de la viabilidad de los contenidos incarcerados, y además aumenta el tiempo quirúrgico de manera significativa, siendo su promedio de 91 minutos.

Actualmente, con el surgimiento de la cirugía robótica, casos reportados como el de Jamshidian, et al. ${ }^{9}$ muestran que es una opción viable para la resolución de la hernia de Spiegel aun con el inconveniente del mayor tiempo quirúrgico y su costo elevado.

En nuestros casos se optó por realizar la técnica IPOM en dos pacientes, uno intervenido de manera electiva y otro como procedimiento de urgencia, ambos con diagnóstico preoperatorio de hernia de Spiegel, diagnosticados de manera oportuna con apoyo de tomografía abdominal. Las dos cirugías se realizaron de manera exitosa, sin complicaciones transoperatorias, posoperatorias ni en su control posterior. Con respecto a la técnica, se vio facilitada de manera sustancial tanto por el tipo de malla como por el aplicador articulado, el cual nos permitió alcanzar de manera más fácil un adecuado ángulo de colocación de los tacks, disminuyendo el tiempo quirúrgico a un promedio de 39 minutos, en contraste con lo reportado para la TAP (45 minutos $)^{16}$ y para la TEP (59 minutos) $)^{5}$. Ambos pacientes presentaron una rápida recuperación, con dolor posquirúrgico mínimo, buen resultado estético y sin complicaciones reportadas.

\section{Conclusión}

El abordaje laparoscópico es el preferido para la reparación de hernias de Spiegel. Dentro de sus técnicas, la IPOM es una opción recomendable por su facilidad técnica y buenos resultados, tanto para cirugías electivas como para cirugías de urgencia. 


\section{Agradecimientos}

Los autores agradecen al departamento de cirugía general del Hospital General Naval de Alta Especialidad por el apoyo brindado.

\section{Responsabilidades éticas}

Protección de personas y animales. Los autores declaran que para esta investigación no se han realizado experimentos en seres humanos ni en animales.

Confidencialidad de los datos. Los autores declaran que han seguido los protocolos de su centro de trabajo sobre la publicación de datos de pacientes.

Derecho a la privacidad y consentimiento informado. Los autores han obtenido el consentimiento informado de los pacientes y/o sujetos referidos en el artículo. Este documento obra en poder del autor de correspondencia.

\section{Bibliografía}

1. Cervantes J, Rojas G, Menéndez A, Cicero A, Vidal P. Hernia de Spiegel. An Med (Mex). 2007;52:65-8.

2. Murillo ZA, Hernández a, Puente J. Hernia de Spiegel. Presentación de un caso y revisión de la literatura. Cirugía endoscópica. 2011;130:130-13.
3. Zaga I, Mayagoitia G. Hernias de pared abdominal. Mayagoitia G (Ed.) Editorial Alfil . 2a. Edición, Ciudad México, México, 2009, pp. 469-74.

4. León S, Acevedo E, Dellepiane V. Hernia de Spiegel, reporte de 23 casos. Revista Chilena de Cirugía. 2011;63: 64-8.

5. Law TT, $\mathrm{Ng} \mathrm{KK}, \mathrm{Ng} \mathrm{L}$, Wong KY. Elective laparoscopic totally extraperitoneal repair for Spigelian hernia: a case series of four patients. Asian J Endosc Surg. 2018;11:244-7.

6. Orthopoulos MD, Kudsi MD. Feasibility of robotic-assisted transabdominal preperitoneal ventral hernia repair. J Laparoendosc Adv Surg Tech A. $2018 ; 28: 434-8$.

7. Colavita PD, Tsirline VB, Walters AL. Laparoscopic versus open hernia repair: outcomes and sociodemographic utilization results from the nationwide inpatient sample. Surg Endosc. 2013;27:109-17.

8. Fernández-Moreno MD, Martí-Cuña MD, Pou G, Ortega J. Intraperitoneal onlay mesh technique for Spigelian hernia in an outpatient and short-stay surgery unit: whats new in intraperitoneal meshes? J Laparoendosc Adv Surg Tech A. 2018;28:700-4.

9. Jamshidian M, Stanek S, Sferra J, Jamil T. Robotic repair of symptomatic Spigelian hernias: a series of three cases and surgical technique review. J Robot Surg. 2018;12:557-60.

10. González A, Escobar E, Romero R, Walker G, Mejías J, Gallas M, et al. Robotic-assisted ventral hernia repair: a multicenter evaluation of clinical outcomes. Surg Endosc. 2017;31:1342-9.

11. Carter JE, Mizes C. Laparoscopic diagnosis and repair of spigelian hernia: report of a case and technique. Am J Obstet Gynecol. 1992;167:77-8

12. Barnes TG, McWhinnie DL. Laparoscopic Spigelian hernia repair: a systematic review. Surg Laparosc Endosc Percutan Tech. 2016;26:265-70.

13. López-Tomassetti Fernández EM, Martín Malagón A, Delgado Plasencia L, Arteaga González I. Laparoscopic repair of incarcerated low Spigelian hernia with transperitoneal PTFE dual mesh. Surg Laparosc Endosc Percutan Tech. 2006;16:427-31.

14. Zacharoulis D, Sioka E. Laparoscopic Spigelian hernia repair: intraperitoneal onlay mesh-plus technique - video presentation. J Laparoendosc Adv Surg Tech A. 2018;28:201-3.

15. Tran H, Tran K, Zajkowska M, Lam V, Hawthorne WJ. Single-incision laparoscopic repair of Spigelian hernia. JSLS. 2015;19:e2015.001644.

16. Barnes TG, McFaul C, Abdelrazeq AS. Laparoscopic transabdominal preperitoneal repair of Spigelian hernia closure of the fascial defect is not necessary. J Laparoendosc Adv Surg Tech A. 2014;24:66-71. 